\title{
Gelsolin in human colon adenocarcinoma cells with different metastatic potential
}

\author{
Monika Litwin ${ }^{1 凶}$, Antonina J. Mazur², Dorota Nowak ${ }^{1}$, Hans G. Mannherz ${ }^{2}$ \\ and Maria Malicka-Błaszkiewicz ${ }^{1}$ \\ ${ }^{1}$ Depratment of Cell Pathology, Faculty of Biotechnology, University of Wroctaw, Wrocław, Poland; ${ }^{2}$ Department \\ of Anatomy and Embryology, Faculty of Medicine, Ruhr-University, Bochum, Germany
}

Received: 18 August, 2009; revised: 19 November, 2009; accepted: 26 November, 2009 available on-line: 04 December, 2009

\begin{abstract}
Gelsolin, one of a major actin-binding proteins, is involved in the regulation of actin cytoskeleton organization by its severing and capping activity towards actin filaments. Human colon adenocarcinoma cell line LS180 and its selected variants of different metastatic potential were used to check for a correlation between gelsolin level, its subcellular localization and the invasive capacity of cells. Based on immunoblotting experiments, a decreased level of gelsolin was detected in the most invasive $5 \mathrm{~W}$ subline when compared to the parental cell line LS180. The intracellular distribution of actin filaments and gelsolin in colon adenocarcinoma cells was examined by confocal microscopy. In the $5 \mathrm{~W}$ subline, unlike in the other examined cells, gelsolin was colocalized with filamentous actin at the cell periphery. In summary, in human colon adenocarcinoma cells, gelsolin level and its subcellular distribution seem to correlate with their metastatic potential.
\end{abstract}

Keywords: gelsolin, actin, cancer

\section{INTRODUCTION}

Changes in cell motility and adhesiveness are observed during tumor growth and seem to be important in determining the metastatic potential and invasiveness of cancer cells (Olson \& Sahai, 2009). Cell motility depends mainly on intracellular organization of the actin cytoskeleton and its dynamic rearrangement, controlled by numerous actin-binding proteins (ABPs), in response to different signals (Pollard \& Borisy, 2003; Dos Remedios et al., 2003). Gelsolin is one of the ABPs having multiple effects on the cellular pool of actin, mainly in regulation of the length of actin filaments. Upon activation by $\mathrm{Ca}^{2+}$ ions or $\mathrm{pH}$ changes, gelsolin severs and caps the fast growing (barbed, +) ends of actin filaments, preventing their elongation. Alternatively, gelsolin can also nucleate actin monomers polymerization. Phosphoinositols (PIP and PIP $_{2}$ ) have been described to release gelsolin from actin filament ends, providing sites for actin assembly (Yin \& Stossel, 1979; Sun et al., 1999). Additionally, gelsolin is involved in several signal transduction pathways through c-erbB-2/ EGFR, Rac and Rho proteins, phosphatidylinositol3-kinase (PI-3K) and phospholipase C (PLC) (Lader et al., 2005).

Actin cytoskeleton organization and the expression level of various ABPs undergo dramatic changes in tumor cells, especially during invasion process (Lambrechts et al., 2004). Gelsolin is widely expressed in normal tissue but altered gelsolin levels have been reported in several types of tumors. Down-regulation of gelsolin has been observed in many transformed cell lines and tumorous tissues, including breast (Dong et al., 2002), colon (Furuuchi et al., 1997), ovarium (Noske et al., 2005), prostate (Lee et al., 1999) and bladder (Tanaka et al., 1995). Gelsolin deficiency increases with progression from

\footnotetext{
${ }^{\square}$ Corresponding author: Monika Litwin, S. Przybyszewskiego 63/77, 51-148 Wrocław, Poland; tel.: (48) 71 375 6206, e-mail: mmach@ibmb.uni.wroc.pl

Abbreviations: ABPs, actin-binding proteins; BSA, bovine serum albumin; EGFR, epidermal growth factor receptor; PBS, phosphate-buffered saline; PIP, phosphatidylinositol 3-phosphate; PIP ${ }_{2}$, phosphatidylinositol 4,5-bisphosphate; TBS, Trisbuffered saline; TRITC, tetramethyl rhodamine isothiocyanate.
} 
benign, non-metastating tumor to invasive breast carcinoma (Winston et al., 2001). Suppression of tumor growth in transformed fibroblasts (Cunningham et al., 1991), breast (Dong et al., 2002), and bladder cancer cells (Rao et al., 2002) was correlated with increased gelsolin expression. However there are studies showing that upregulation of gelsolin significantly correlates with worse prognosis and higher risk of cancer recurrence in cases of nonsmall cell lung carcinoma (Yang et al., 2004). Low level of gelsolin at early stages of malignant transformation was observed by Rao and coworkers (2002) in urothelial carcinomas, whereas increased gelsolin expression accompanied the transition from non-invasive to invasive state of this type of cancer. Several studies have shown a relation between gelsolin level and cell migration ability. In fibroblasts, overexpression of gelsolin resulted in reduction of actin filaments, followed by decreased cell adhesion ability and increased motility (Cunningham et al., 1991). Although the structure and function of gelsolin have been well characterized, its role in cancer cell invasion remains unclear and published data are controversial.

Our previous results have shown a distinct correlation between the metastatic potential of human colon adenocarcinoma cell lines and increased level of $\beta$-actin (Nowak et al., 2005). In this paper, the same colon adenocarcinoma parental cell line (LS180) and selected variants (EB3, 3LNLN, 5W) were used to observe whether gelsolin level and distribution correlate with the metastatic potential in respect to the actin cytoskeleton organization.

\section{MATERIALS AND METHODS}

Cell culture. Human colon adenocarcinoma parental cell line LS180 and its selected variants characterized with different metastatic capacity (EB3, 3LNLN and $5 \mathrm{~W}$ ) were obtained from the Institute of Immunology and Experimental Therapy, Polish Academy of Sciences (Wrocław, Poland). The parental cells (LS180) were selected and characterized by Opolski and coworkers (1998). In vitro selection of the LS180 cell line resulted in the subline named EB3, of the highest migration ability. Cells selected in vitro were then selected in vivo for tumor formation in lymph nodes, where by the 3LNLN cell subline was isolated. Highly metastatic $5 \mathrm{~W}$ subline was obtained from tumor metastasis to the liver. The details on the selection procedure are given in our prevoius paper (Nowak et al., 2002). The metastatic capacity of the parental adenocarcinoma cell line LS180 and the selected variants was additionally confirmed by invasive factor measurements on Matrigel-coated Transwell filters.
Cells were grown in OptiMEM medium supplemented with 5\% fetal bovine serum (FBS) (Gibco, Germany). Cells were cultured at $37^{\circ} \mathrm{C}$ under a humidified atmosphere of $5 \% \mathrm{CO}_{2}$.

Preparation of cellular extracts. Cellular extracts were prepared for gelsolin level determination. Cells grown to confluence on culture dishes were washed twice with PBS, scraped with a cell scraper, suspended in PBS and centrifuged (5 min, $\left.2655 \times g, 4^{\circ} \mathrm{C}\right)$. The cell pellets were treated with lysis buffer $(10 \mathrm{mM}$ Tris/ $\mathrm{HCl} \mathrm{pH} 7.8,1 \mathrm{mM}$ EDTA, $1 \mathrm{mM} \mathrm{MgCl}, 0.02 \%$ Triton X-100) with proteinase inhibitors (Sigma Aldrich, Germany). Thereafter the samples were sonicated for $30 \mathrm{~s}$ at $70 \%$ force with an Ultra-Turrax T8 device, centrifuged $\left(10000 \times g, 30 \mathrm{~s}, 4^{\circ} \mathrm{C}\right)$ and supernatants were used for immunoblotting analysis. Protein concentration was determined by the Bradford procedure (Bradford, 1976) with bovine serum albumin (BSA) as a standard.

Western blot analysis. Proteins $(25 \mu \mathrm{g})$ were separated by polyacrylamide gel electrophoresis in the presence of sodium dodecylsulfate (SDS/ PAGE) according to Laemmli (1970), followed by transfer to nitrocellulose membrane by the procedure of Towbin et al. (1979). The membrane was blocked with a $5 \%$ non-fat dried milk solution in $0.1 \%$ Tween-20 in TBS. Polyclonal rabbit anti-gelsolin antibodies, obtained as described by Hestercamp et al. (1993) were used for gelsolin identification. $\beta$-Tubulin, labelled by mouse anti- $\beta$-tubulin antibody (Sigma-Aldrich), was used as an internal loading control in parallel. Secondary antibodies were applied according to the manufacturer's protocols: horseradish peroxidase (HRP)-conjugated goat anti-mouse IgG (Cell Signalling) and goat anti-rabbit IgG HRP-linked (Cell Signalling). The nitrocellulose membranes were developed by enhanced chemiluminescence (ECL) system (Amersham Bioscience). The intensity of bands was quantified by densitometry using Quantity One software (Gel Doc1000/2000, Bio-Rad) and expressed as integrated optical density (IOD).

Statistical analysis. All data are reported as mean \pm standard deviation (S.D.). The significance of data was determined by Student's $t$-test (significance level was set at $P<0.05$ ).

Confocal microscopy. The subcellular distribution of actin filaments and gelsolin in colon adenocarcinoma cells sublines was examined by fluorescence staining in an inverted confocal laser scanning microscope (LSM510, Zeiss, Jena, Germany). Cells grown on sterile glass coverslips were fixed with $4 \%$ paraformaldehyde (PFA) for $20 \mathrm{~min}$, permeabilized with $0.1 \%$ Triton X-100 in PBS for 3 min, washed three times with PBS and incubated 


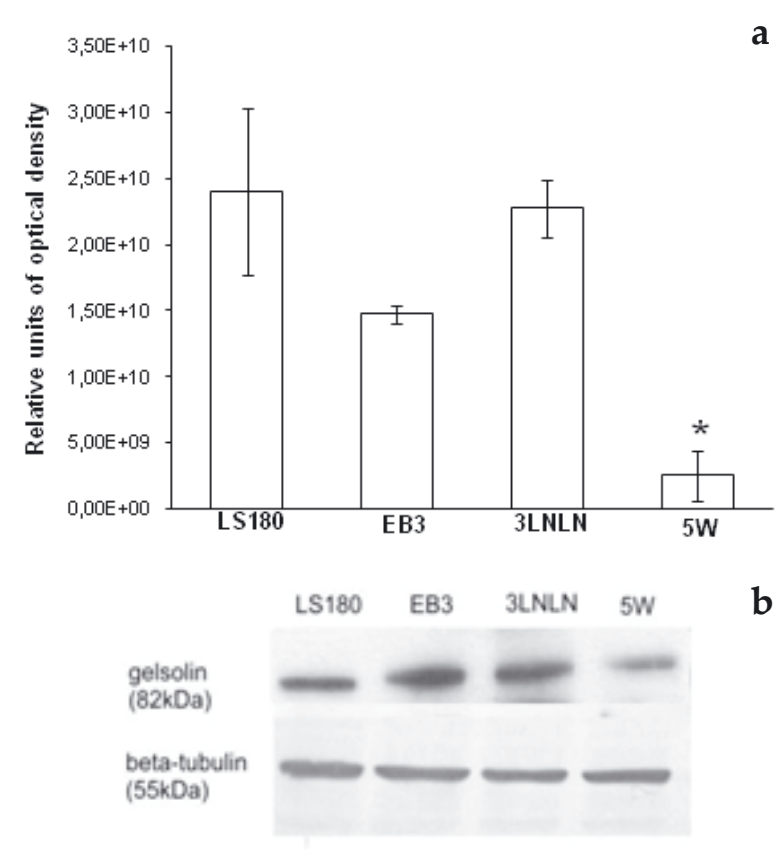

Figure 1. Gelsolin level in human colon adenocarcinoma cells.

a) Densitometric analysis of gelsolin level in cellular extracts from human colon adenocarcinoma LS180 cells and their selected variants of different metastatic potential. Results are expressed as units of integrated optical density (IOD). *Indicates value significantly different from that for parental line (LS180) as determined by Student's $t$-test; significance level was set at $P<0.05$. Bars represent the mean $\pm S$.D. from four independent experiments. b) Representative immunoblot of gelsolin in colon adenocarcinoma cell lines. $\beta$-Tubulin was used as internal loading control.

with 1\% BSA in PBS for 30 min. Rabbit anti-gelsolin antibodies (1:500 dilution) and goat anti rabbitAlexaFluor 488 (1:200 dilution, Molecular Probes ${ }^{\mathrm{TM}}$, Invitrogen) were applied to visualize gelsolin. Actin filaments were stained with TRITC-labelled phalloidin $(0.4 \mu \mathrm{g} / \mathrm{ml}$, Sigma-Aldrich). About 25 cells were photographed every time in three independent experiments and representative cells of every subline are presented.

\section{RESULTS}

\section{Gelsolin level}

Gelsolin levels were analyzed in cellular extracts prepared from human colon adenocarcinoma parental cell line (LS180) and selected variants (EB3, 3LNLN, 5W). Quantitative densitometric immunoblot analysis showed a statistically significant decrease in the gelsolin level in the $5 \mathrm{~W}$ subline selected in vivo and characterized by the highest invasive capacity compared with the parental LS180 line and other sublines (Fig. 1).

\section{Gelsolin distribution and actin cytoskeleton organi- zation}

The lower level of gelsolin in the most invasive $5 \mathrm{~W}$ subline led our attention to its subcellular distribution in relation to the actin cytoskeleton organization. In the parental human colon adenocarcinoma LS180 cells and their selected variants actin was organized as a meshwork in the cell body. Only in the LS180 cells short actin stress fibers were observed (Fig. 2, panel I, long arrows). In the cell variants of rounded morphology (EB3 and 5W) actin was concentrated under the cell membrane as a cortical ring (Fig. 2, panel I, short arrows). Microscopic observations of the cells immunostained with anti-gelsolin antibodies revealed that gelsolin was dispersed within the whole cell body in the parental cell line LS180 as well as in the EB3 and 3LNLN sublines. In contrast, in the highly invasive $5 \mathrm{~W}$ subline gelsolin was concentrated under the plasma membrane and strongly colocalized with filamentous actin (Fig. 2, panel III, arrowheads).

\section{DISCUSSION}

Our previous studies (Nowak et al., 2002) have demonstrated that there is a direct correlation between the metastatic capacity of human colon adenocarcinoma cells selected in vitro and in vivo and the state of actin polymerization measured as filamentous $(\mathrm{F})$ to monomeric $(\mathrm{G})$ actin ratio (F: G). It should be underlined that the highest value of this ratio was seen in the $5 \mathrm{~W}$ subline, characterized by the highest metastatic capacity in comparison with the parental LS180 cells and EB3 selected in vitro and 3LNLN selected in vivo (Nowak et al., 2002). Actin polymerization and cytoskeleton organization are affected by numerous regulatory proteins (ABPs). Our attention has been focused on gelsolin, an actin-binding protein involved in the regulation of actin polymerization by severing and capping actin filaments (Sun et al., 1999). There are studies indicating a potential role of gelsolin during invasion of cancer cells. Some data suggest that gelsolin level oscillates during the metastasis process in different types of tumor cells (Lambrechts et al., 2004). Our current experimental data show a statistically significant decreased gelsolin level in the $5 \mathrm{~W}$ subline, accompanied by its distinct subcellular distribution. Although some studies show a role of gelsolin during migration of cancer cells, its subcellular redistribution connected with changes in the protein level have not been observed until now. The decreased level of gelsolin as well as its relocation to the peripheral area of the cell can lead to the more invasive phenotype and the most malignant features of the 
I II

LS180
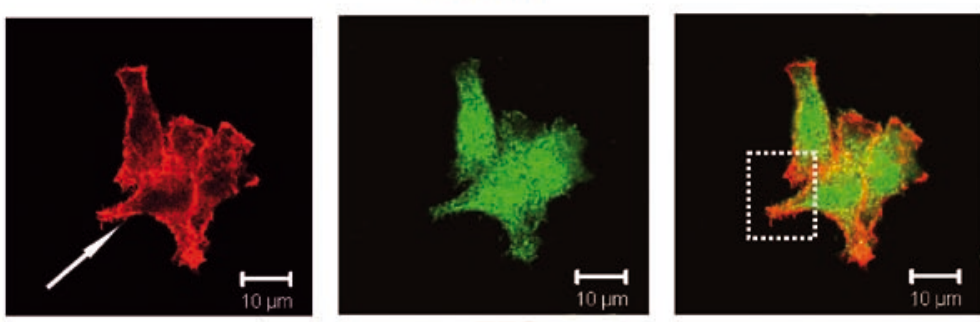

EB3
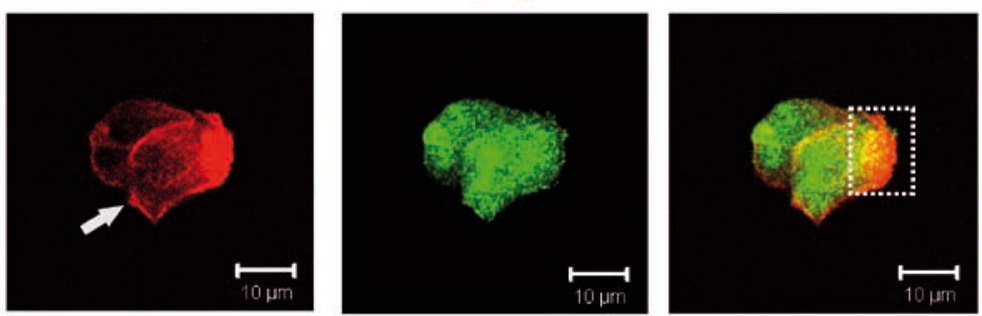

3LNLN
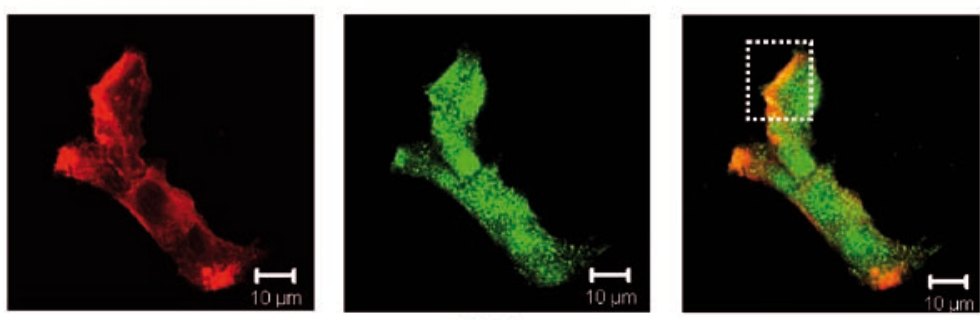

$5 \mathrm{~W}$
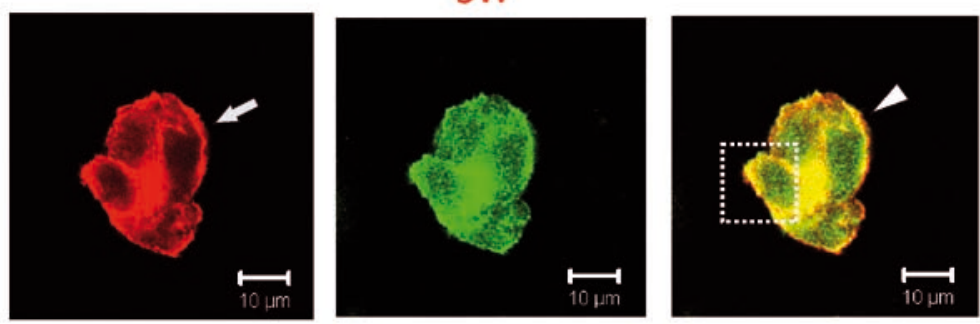
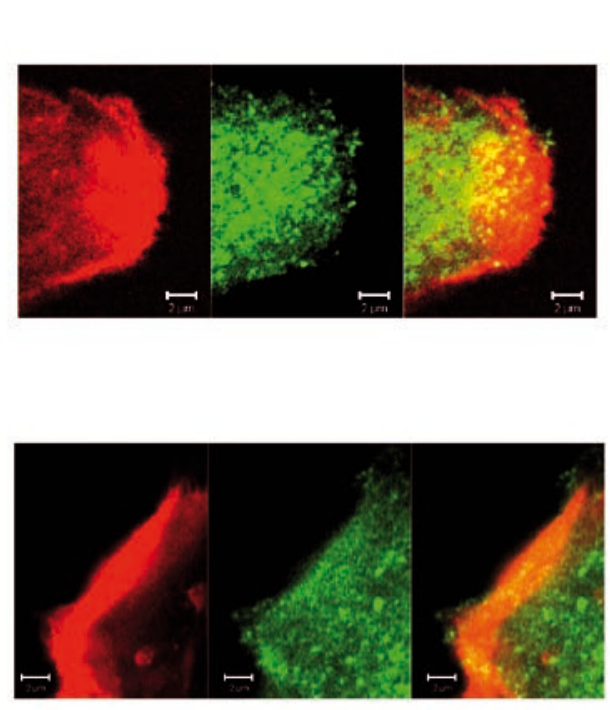

IV
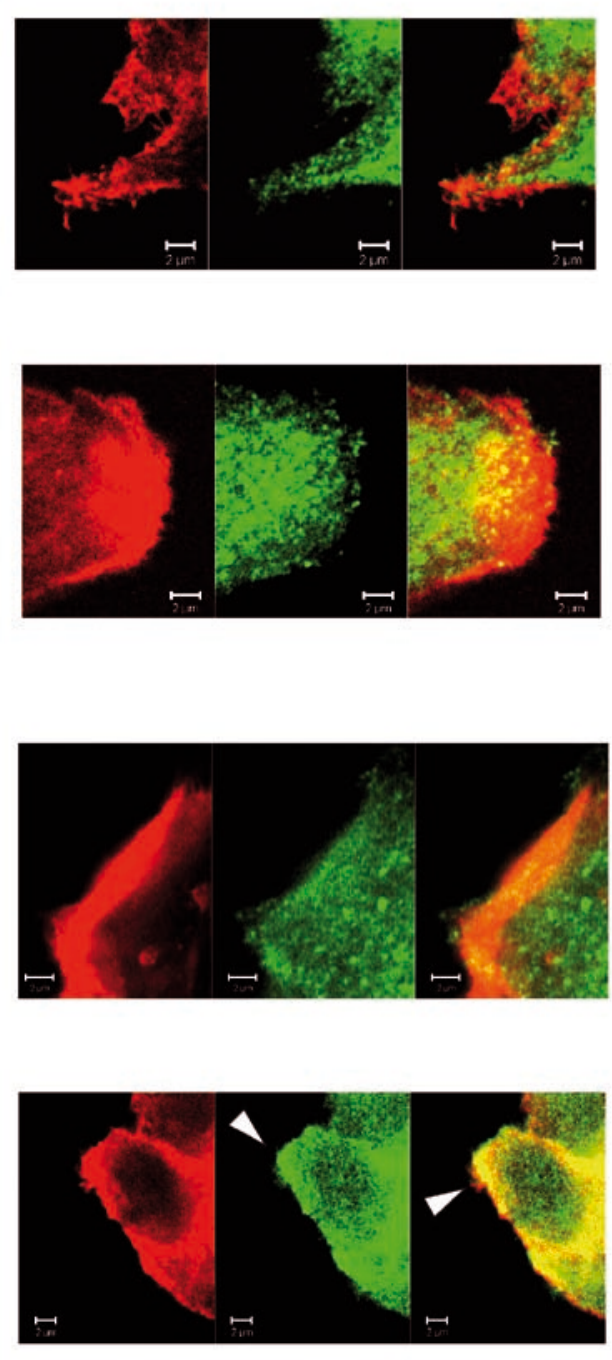

Figure 2. Subcellular localization of gelsolin and filamentous actin organization in LS180 cells and selected variants. Insets represent zoomed area marked with dotted line. Bars correspond to $10 \mu \mathrm{m}$ and to $20 \mu \mathrm{m}$ in insets. Panel I. Actin filaments stained with TRITC-labelled phalloidin (red). Panel II. Immunostaining for gelsolin (green). Merged images are depicted in the right column (Panel III). Panel IV. insets. Long arrows: stress fibers, short arrows: submembrane F-actin, arrowheads: colocalization of gelsolin and filamentous actin under cellular membrane.

5W subline. Differences in cell morphology between the sublines of human colon adenocarcinoma cells (shown in Fig. 2) have been observed previously by Nowak et al. (2005). Several studies have been performed to elucidate the mechanism underlying the different migration modes of cancer cells: ameboid for rounded cells and mesenchymal for elongated ones. Each type of locomotion is connected with diverse requirements for actin binding proteins, Rho family GTPases and extracellular proteolysis (Wolf et al., 2003). Cytoplasmic $\beta$-actin seems to play a crucial role in the process of movement, required for intravasation of cancer cells through the vessel walls and for metastasis (Peckham et al., 2001; Popow et al., 2006). An increased expression of cytoplasmic $\beta$ actin was found in selected human adenocarcinoma sublines as compared with the parental LS180 cells (Nowak et al., 2005). Our studies of human colon adenocarcinoma cancer sublines - selected in vitro and in vivo and characterized with different metastatic potential confirm the importance of gelsolin in the migration of colon cancer cells. We assume that in the $5 \mathrm{~W}$ subline, characterized with the highest invasive potential, the lowered gelsolin level and its subcellular redistribution are somehow related with the high migration capacity of these cells. However, 
since different cells migrate in diverse ways in response to various signaling pathways, the significance of gelsolin expression and localization in relation to cancer cell invasiveness may be strongly dependent on the cell type and other internal and external factors.

\section{REFERENCES}

Bradford MM (1976) A rapid and sensitive method for the quantitation of microgram quantities of protein utilizing the principle of protein-dye binding. Anal Biochem 72: $248-254$.

Cunningham CC, Stossel TP, Kwiatkowski DJ (1991) Enhanced motility in NIH 3T3 fibroblasts that overexpress gelsolin. Science 215: 1233-1236.

Dong Y, Asch HL, Ying A, Asch BB (2002) Molecular mechanism of transcriptional repression of gelsolin in human breast cancer cells. Exp Cell Res 276: 328-336.

Dos Remedios CG, Chhabra D, Kekic M, Dedova IV, Tsubakihara M, Berry DA, Nosworthy NJ (2003) Actin binding proteins: regulation of cytoskeletal microfilaments. Physiol Rev 83: 433-473.

Furuuchi K, Fujita H, Tanaka M, Shichinohe T, Senmaru N, Ogiso Y, Moriya S, Hamada M, Kato H, Kuzumaki N (1997) Gelsolin as a suppressor of malignant phenotype in human colon cancer. Tumor Target 2: 277-283.

Hesterkamp T, Weeds AG, Mannherz HG (1993) The actin monomers in the ternary gelsolin: 2 actin complex are in an antiparallel orientation. Eur J Biochem 218: 507-513.

Lader AS, Lee JJ, Cicchetti G, Kwiatkowski DJ (2005) Mechanism of gelsolin-dependent and -independent EGF-stimulated cell motility in human lung epithelial cell lines. Exp Cell Res 307: 153-163.

Laemmli UK (1970) Cleavage of structural proteins during the assembly of the head of bacteriophage T4. Nature 15: 680-685.

Lambrechts A, Van Troys M, Ampe C (2004) The actin cytoskeleton in normal and pathological cell motility. Int J Biochem Cell Biol 36: 1890-1909.

Lee HK, Driscoll D, Asch H, Zhang PJ (1999) Downregulation of gelsolin expression in hyperplastic and neoplastic lesion of the prostate. Prostate 40: 14-19.

Mullauer L, Fujita H, Ishizaki A, Kuzumaki N (1993) Tumor-suppressive function of mutated gelsolin in rastransformed cells. Oncogene 8: 2531-2536.

Noske A, Denkert C, Schober H, Sers C, Zhumabayeve B, Wiechert W, Dietel M, Wiechen K (2005) Loss of gelsolin expression in human ovarian carcinomas. Eur J Cancer 41: 461-469.

Nowak D, Krawczenko A, Duś D, Malicka-Błaszkiewicz M (2002) Actin in human colon adenocarcinoma cells with different metastatic potential. Acta Biochim Polon 49: 823-828.
Nowak D, Skwarek-Maruszewska A, Zemanek-Zboch M, Malicka-Błaszkiewicz M (2005) Beta-actin in human colon adenocarcinoma cell lines with different metastatic potential. Acta Biochim Polon 52: 461-468.

Olson MF, Sahai E (2009) The actin cytoskeleton in cancer cell motility. Clin Exp Metastasis 26: 273-287.

Opolski A, Wietrzyk J, Duś D, Kieda C, Matejuk A, Makowska A, Wojdat E, Ugorski M, Laskowska A, Kłopocki A, Rygaard J, Radzikowski C (1998) Metastatic potential and saccharide antigens expression of human colon cancer cells xenotransplanted into athymic nude mice. Folia Microbiol 43: 507-510.

Peckham M, Miller G, Wells C, Zicha D, Dunn GA (2001) Specific changes to the mechanism of cell locomotion induced by overexpression of beta-actin. J Cell Sci 114: 1367-1377.

Pollard TD, Borisy G (2003) Cellular motility driven by assembly and disassembly of actin filaments. Cell 112: 453-465.

Popow A, Nowak D, Malicka-Blaszkiewicz M (2006) Actin cytoskeleton and beta-actin expression in correlation with higher invasiveness of selected hepatoma Morris 5123 cells. J Physiol Pharmacol 57: 111-123.

Rao J, Seligson D, Visapaa H, Horvath S, Eeva M, Michel K, Pantuck A, Belldegrun A, Palotie A (2002) Tissue microarray analysis of cytoskeletal actin associated biomarkers gelsolin and E-cadherin in urothelial carcinoma. Cancer 95: 1247-1257.

Sun HQ, Yamamoto M, Mejillano M, Yin HL (1999) Gelsolin a multifunctional actin regulatory protein. J Biol Chem 274: 33179-33182.

Tanaka M, Mullauer L, Ogiso Y, Fujita H, Moriya S, Furuuchi K, Harabayashi T, Shinohara N, Koyanagi T, Kuzumaki N (1995) Gelsolin a candidate for suppressor of human bladder cancer. Cancer Res 55: 3228-3232.

Towbin H, Staechelin T, Gordon J (1979) Electrophoretic transfer of proteins from polyacrylamide gels to nitrocellulose sheets: procedure and some applications. Proc Natl Acad Sci USA 76: 4350-4355.

Winston JS, Asch HL, Zhang PJ, Edge SB, Hyland A, Asch BB (2001) Downregulation of gelsolin correlates with the progression to breast carcinoma. Breast Cancer Res Treat 65: 11-21.

Wolf K, Mazo I, Leung H, Engelke K, Andrian UH, Deryugina EI, Strongin AY, Borcker EB, Friedl P (2003) Compensation mechanism in tumor cell migration: mesenchymal-amoeboid transition after blocking of pericellular proteolysis. J Cell Biol 160: 267-277.

Yang J, Tan D, Asch H, Swede H, Bepler G, Geradts J, Moysich K (2004) Prognostic significance of gelsolin expression level and variability in non-small cell lung cancer. Lung Cancer 46: 29-42.

Yin HL, Stossel TP (1979) Control of cytoplasmic actin gelsol transformation by gelsolin a calcium-dependent regulatory protein. Nature 281: 583-586. 